\title{
Kernos
}

Revue internationale et pluridisciplinaire de religion grecque antique

$13 \mid 2000$

Varia

\section{Jean RUDHARDT, Thémis et les Hôrai. Recherche sur les divinités grecques de la justice et de la paix}

\section{Danièle Aubriot-Sévin}

\section{OpenEdition \\ Journals}

Édition électronique

URL : http://journals.openedition.org/kernos/1332

DOI : 10.4000/kernos.1332

ISSN : 2034-7871

\section{Éditeur}

Centre international d'étude de la religion grecque antique

Édition imprimée

Date de publication : 1 janvier 2000

ISSN : 0776-3824

\section{Référence électronique}

Danièle Aubriot-Sévin, « Jean RUDHARDT, Thémis et les Hôrai. Recherche sur les divinités grecques de la justice et de la paix », Kernos [En ligne], 13 | 2000, mis en ligne le 16 juin 2011, consulté le 24 septembre 2020. URL : http://journals.openedition.org/kernos/1332 ; DOI : https://doi.org/10.4000/ kernos. 1332 
retenu dans son Corpus la «Suite pythique » de l'Hymne pseudo-homérique à Apollon.

Enfin, quelques broutilles d'usage : p. 13, 1. 1, il fallait écrire "au meilleur de son combat ... "; p. 194 et 195, " Areopago »; p. 199, "Wilamowitz-Moellendorff " et p. 203, ajouter entre Reichel et Rose une référence à la Psyche d'Erwin Rohde.

Pierre Somville

(Université de Liège)

Jean RudhardT, Thémis et les Hôrai. Recherche sur les divinités grecques de la justice et de la paix, Genève, Droz, 1999. 1 vol. $15 \times 22 \mathrm{~cm}, 167$ p. (Recherches et Rencontres. Publications de la Faculté des lettres de Genève, 14). ISBN : 2-600-00354-1.

Il faut saluer la publication du nouveau livre magistral de Jean Rudhardt. Ce volume, consacré à ce qu'on appelait auparavant des notions divinisées, met en évidence que Thémis, Eunomia, Dikè, et Eirènè ne sont pas des personnifications abstraites ou des allégories : l'auteur, se fiant à ce qu'exprime le langage mythique en insérant Thémis et les Hôrai dans une généalogie, et prenant acte de la constatation que ces entités recevaient un culte, montre que ce sont en fait de vraies divinités (qui ne sont pas le produit d'une invention tardive), et sonde le sentiment du divin qui se laisse déchiffrer à travers elles. C'est-à-dire que, dans la droite ligne d'une réflexion commencée depuis plusieurs décennies, il nous fait progresser dans la "compréhension » de la pensée religieuse grecque, dont le sens a longtemps échappé malgré les connaissances qu'on pouvait avoir à son sujet.

Dès son premier ouvrage - si bien nommé : Notions fondamentales de la pensée religieuse et actes constitutifs du culte, Genève, 1958 (réimprimé à Paris en 1992) -, Jean Rudhardt s'est imposé d'emblée comme l'un des spécialistes les plus perspicaces de la religion grecque. Suivant un système original, il s'attachait à appréhender les faits religieux tels qu'ils furent vécus à l'intérieur d'une société limitée dans l'espace et dans le temps, en essayant de rétablir en nous le contexte psychologique dans lequel venait s'inscrire la conduite d'un fidèle à l'occasion d'une circonstance concrète. C'est donc une méthode interne, qui vise à comprendre dans une même démarche, en une alliance significative, à la fois le culte et la pensée. La condition pour atteindre à pareille connivence intime entre le lecteur moderne et les textes anciens est de faire autant que possible table rase de nos modes de pensée, et en tout cas d'ordonner les faits hétérogènes rencontrés selon les catégories que la langue grecque nous propose. Aussi, dans la certitude que ces faits ne sauraient nous être exactement intelligibles hors de la langue employée par les hommes qui les ont vécus, l'A. part-il des textes pour y revenir sans cesse. Cette exigence de rigueur portée à un degré extrême dans des ouvrages dont le but immédiat n'est pas d'être une pure recherche sur le vocabulaire distingue les travaux de Jean Rudhardt de tous les autres, et fait de leur auteur un maître incontesté en matière de religion grecque. Les analyses lexicologiques et la lecture attentive des textes qui formaient déjà le socle de son premier livre ont constitué la base de tous les suivants jusqu'à celui-ci. C'est ainsi que la carrière de Jean Rudhardt n'a été jalonnée que d'ouvrages fondamentaux. Après la pensée religieuse et les actes du culte, la même méthode a en effet été appliquée par l'A. aux mythes (principalement cosmogoniques), en particulier dans deux livres ( $L e$ thème de l'eau primordiale dans la mytbologie grecque, Berne, 1971; Le rôle d'Éros et d'Aphrodite dans les cosmogonies grecques, Paris, 1986), ainsi que dans de nombreux articles, dont quelques-uns des plus anciens ont été réunis en 1981 
en un volume, lui aussi fondateur, intitulé $D u$ mythe, de la religion grecque et de la comprébension d'autrui, Revue européenne des sciences sociales, XIX (Cahiers Vilfredo Pareto). S'y trouvent rassemblées entre autres des études montrant comment le mode d'expression mythique fait partie intégrante de la pensée religieuse grecque. De multiples articles ont suivi, approfondissant ces considérations, et les élargissant de plus en plus à la tradition orphique. Avec Thémis et les Hôrai, l'A. s'attaque à des divinités réputées abstraites auxquelles un moderne répugne à accorder crédit religieux - plus encore que lorsqu'il s'agit d'autres figures du panthéon -, et ce faisant, il nous plonge en fait au cœur même de la religion grecque.

Après une substantielle introduction méthodologique, le livre se présente en trois chapitres : 1) Thémis; 2) les Hôrai; 3) traits particuliers de chacune des Hôrai (Eunomia, Dikè, Eirènè). Toute l'analyse est conduite selon l'idée directrice fondamentale que ce sont les mêmes noms qui, désignant certaines circonstances où les Grecs perçoivent les effets d'une action divine, désignent du même coup le divin dont elles signifient la présence - légitimation irrécusable, s'il en était besoin, de l'attention méticuleuse consacrée au vocabulaire dans une étude de notions religieuses.

Au premier chapitre, un examen des emplois du nom thémis, de son pluriel themistes, ainsi que de ses dérivés (verbes et adjectifs) amène à la récapitulation suivante : "La thémis... est une exigence ressentie, le désir ou le besoin d'inventer des conduites propres à assurer une entente, ... à assurer la paix et l'équilibre d'une société..., tandis que les actes contraires à la thémis dissolvent les sociétés... " Passant du nom commun au nom divin, on rencontre une Titanine qui, n'ayant pas partagé l'exil de ses frères et de ses autres sœurs après la guerre avec les Cronides, garde une efficacité discrète à l'intérieur du monde et est liée à Zeus, dont (avec Mètis et Héra) elle est l'une des épouses. Le sens de ces péripéties mythologiques est bien dégagé : par ce mariage, le dieu souverain attache à sa personne à la fois un passé proche des origines, et les aspirations morales incarnées en Thémis - bel exemple du lien entre expression mythique et pensée religieuse. Inversement, la déesse agit sous le contrôle de Zeus pour contribuer à l'ordre du monde qu'il garantit: liée au pouvoir prophétique, gardienne des portes célestes autant que patronne des assemblées, elle concourt au fonctionnement des institutions (divines comme humaines), ainsi qu'à la résolution des conflits. Aussi l'importance de Thémis n'est-elle pas des moindres : quoique l'action de la déesse s'exerce rarement d'une manière immédiate parmi les hommes - tout comme la notion de la thémis, perceptible à travers les conduites humaines qu'elle guide, est difficile à conceptualiser -, Thémis ne laisse pas de recevoir un culte, volontiers en association avec d'autres dieux; c'est que l'influence qu'elle exerce sur les choses est indirecte, car elle inspire ou assiste d'autres divinités plus actives. En effet, de même que «la thémis n'est pas la règle établie mais l'exigence qui conduit à la découverte d'une règle et à son observation, Thémis de son côté n'est pas la loi mais la cause de la loi » (p. 56). Jean Rudhardt propose de traduire Thémis par «Exigence d'équilibre ».

Le deuxième chapitre est consacré aux Hôrai ou plutôt, selon la même méthode que pour le premier, d'abord au nom commun bôra et à deux adjectifs ( $\dot{\omega} p a \hat{o} o s$ et $\ddot{p} p l o s)$ qui en dérivent (et qui viennent confirmer les observations faites à propos du nom); dans un deuxième temps sont considérées les divinités. Son caractère volontiers périodique, son aptitude à évoquer « des conditions propices à un développement, à une croissance, à une maturation (ces conditions pouvant être éventuellement temporelles ou locales » (p. 70), et ainsi sa vocation à désigner un temps qui convient aux fêtes religieuses, font que l'bôra, quoique le 
plus souvent unie à une notion de durée, n'en est cependant pas nécessairement tributaire. Proche de kairos à certains égards, elle s'en distingue en ce qu'elle procède de la nature. Les Hôrai quant à elles sont un groupe de déesses nées de Zeus et de Thémis, et possédant en plusieurs lieux statues et autels; si les traditions divergent quant à leur nombre et quant au nom porté par chacune d'entre elles (les Athéniens en particulier ont parfois avancé le chiffre de deux), c'est le chiffre hésiodique de trois qui s'est imposé, ainsi que les noms Eunomiè (Bonne organisation), Dikè (Justice) et Eirènè (Paix), qui désignent clairement en elles des divinités politiques. Leur étude individuelle montre qu'elles assurent ensemble le succès des activités sociales et la prospérité d'une cité, mais que leur domaine ne s'épuise pas dans les bornes de la vie civique : liées à la floraison, à l'amour, à la séduction, ainsi qu'aux mouvements sidéraux, proches de Perséphone et d'Aphrodite, elles participent aux rondes divines, et présentent d'étroites affinités avec les puissances obscures qui président à toutes les formes de croissance naturelle. Ces forces sont perçues dans le déroulement du processus auquel elles contribuent et associées volontiers à la durée de son accomplissement. Plus encore que leur mère Thémis donc, elles affectent à la fois la vie des sociétés humaines et la vie du cosmos, que la religion grecque ne sépare pas. Honorer "bonne organisation ", "justice " et "paix » sous le nom de Hôrai re vient à signifier qu'elles sont tenues pour divines : là encore, le mode d'expression myhique vient relayer la difficulté à conceptualiser ces divinités.

Le troisième chapitre enfin considère les traits particuliers de chacune des Hôrai - dont la mythologie est relativement pauvre, surtout pour deux d'entre elles. L'auteur situe eunomia sur un plan très général : au-delà de l'institution sociale comme au-delà de l'individu, au-delà de l'ordonnance de la société, de l'équilibre interne de la personne humaine, elle affecte le principe qui régit l'harmonie (quelle que soit son application). Comme ce principe est « tenu pour divin à l'instant même où l'on en ressent les effets » (p. 102), Eunomia est une déesse qui, patronne de plusieurs villes, est vénérée sur des autels un peu partout selon Démosthène. Même Eirènè, dont on serait en peine de décrire des cultes, était néanmoins l'objet de plusieurs rites. Jean Rudhardt constate qu'il est parfois difficile, à l'intérieur d'un même texte, de discerner si le mot eirènè est en rapport avec l'état de paix, ou avec une figure anthropomorphe, tant il est vrai que les auteurs, "percevant la paix, source de mille bienfaits, comme merveil leuse, (voient) simultanément en elle un état de fait et une divinité » (p. 148). Il n'en va pas autrement pour le mot dikè qui " paraît désigner tantôt la justice dont l'homme sent obscurément qu'elle se manifestera sous la forme de récompenses ou de châtiments, tantôt une déesse dotée de pensée et de volontés » (p. 133). Mais le cas de Dikè est infiniment plus riche et plus complexe que celui de ses sœurs, et c'est d'elle que les textes nous parlent le plus souvent. Aussi le développement qui lui est réservé occupe-t-il la plus grande place de ce troisième chapitre. Il est montré que, contrairement à ce qu'affirmait Glotz, la dikè peut être familiale, comme la thémis; et qu'elle désigne le lot convenant à un individu, en considération des actes qu'il a accomplis - le mot semblant « énoncer l'idée d'une convenance souhaitable entre (une) action (réalisée) et les circonstances de son exécution; entre cette action et l'homme ou la femme qui en sont l'objet; entre la personne agissante et certains effets indirects des gestes qu'elle (effectue) » (p. 111). En somme, ce nom lie un acte à ses justes conséquences, ce que

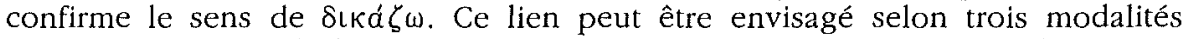
suivant qu'on regarde la dikè comme une force agissant sur le cours des événements..., qu'on parle de la dikè subjective telle qu'elle influence les conduites humaines, ou que l'on considère la justice telle que des juges ou des tribunaux l'exercent - et c'est bien cet ordre qui à l'auteur paraît le plus conforme à la 
mentalité grecque, en ce qu'il situe l'action mystérieuse de la justice dans l'histoire avant la pratique sociale (pourtant réalité plus immédiatement saisissable pour un esprit moderne). Suit une fine étude des autres mots apparentés, avant d'en venir aux trois sœurs, qui ont chacune des fonctions ou des qualités propres, et disposent chacune d'une part d'initiative : la dépendance filiale des Hôrai par rapport à Thémis est une nouvelle illustration de ce que l'auteur a eu mainte occasion de montrer ailleurs, i.e. que «les qualités confondues dans un ancêtre s'expriment (d'une manière plus individualisée) dans la personne de ses descendants » (p. 49).

Ce rapide survol voudrait faire deviner quelque chose de l'extraordinaire profondeur de ce livre. Mais il n'a aucune chance de laisser concevoir la finesse des analyses précises et très neuves sur lesquelles est fondé cet ouvrage. C'est ainsi que sont mis en place les rapports respectifs de $\delta(\kappa \eta, \delta i \kappa a \iota \nu, \delta \iota \kappa a \iota \sigma u ́ v \eta$ (p. 125 sq.), que sont examinées les relations de $\delta(\kappa \eta$ et de aloẃs (p. $131 s q$.), de

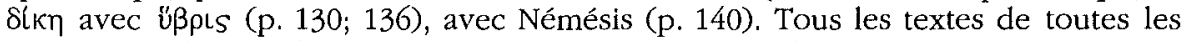
périodes et de tous les genres sont convoqués et sondés, d'Homère aux Hymnes orphiques, des Présocratiques à Platon ou à Xénophon, et à l'occasion jusqu'au grec moderne. Aussi bien Jean Rudhardt est-il même amené, chemin faisant, à modifier des traductions (p. 72-3, pour le Phédon et l'Axiocbos), à élucider des endroits difficiles (p. 93 : Pd. Ol. IV, 1 sq.; p. 119 sq. : Hés. TJ., 9) ou mal compris (p. 111-2: Od. XVIII, 275), à préciser ou à nuancer des passages ambigus (p. 116 : Il. XIX, 180). Ce ne sont là que quelques exemples : de toutes ces richesses, même un aperçu ne saurait être procuré ici.

Si la reconsidération des rapports entre Thémis et Dikè constitue un apport de premier plan pour les hellénistes, elle est utile aussi pour les autres disciplines de l'antiquité. Glotz distinguait, comme on sait (p. 15), entre justice familiale, fermement établie, appelée themis $(\theta \dot{\mu} \mu \mathrm{s})$, et justice interfamiliale, plus fluide, que désignerait le nom dikè ( $\delta i ́ k \eta$ ), et cette distinction n'avait guère été remise en cause. Benveniste pour sa part mettait l'accent sur une opposition, pour dikè et thémis, entre le droit humain et le droit divin (p. 130). Jean Rudhardt revient sur ces propositions et, sans se prononcer catégoriquement sur l'étymologie de $\theta \dot{\epsilon} \mu \mathrm{s}$ il suggère, si l'on peut retenir pour ce nom le même radical que pour $\tau(\theta \eta \mu \iota$, d'être sensible à une possible valeur active de la racine verbale : "La thémis ne serait pas ce qui est placé, définitivement mis en place mais ce qui met en place ou contribue à le faire » (p. 26). Il observe que l'examen des textes anciens ne confirme pas la classification de Glotz, pas plus qu'il ne permet de s'arrêter à celle de Benveniste. À ses yeux, "alors que la thémis est universelle, même si elle impose des exigences variables selon les circonstances, la dikè se définit d'emblée en considération de qualités particulières aux personnes qu'elle concerne " (p. 108). Toutefois, si dikè peut être subjective, elle peut également posséder une valeur normative par quoi elle est aussi objective que thêmis, mais (si l'on peut dire en reprenant le langage mythique) la " mère " s'exprime en principes universels, en préceptes traditionnels, tandis que la «fille", à la fois plus contraignante et plus concrète, est présente à travers les réactions effectives des hommes à ces principes. C'est-à-dire que « si la thémis précède l'action humaine, la dikè lui succède... La thémis est une exigence universelle; de l'ordre de l'argumentation, les thémistes ont un caractère théorique. Concernant des individus particuliers, les dikai sont immédiatement pratiques. Elles sont exécutoires » (p. 129). Comme on le voit, la correction qu'impose l'étude circonstanciée des textes à une opinion acceptée depuis un siècle est d'importance, et doit intéresser non seulement historiens des religions et philologues, mais encore philosophes et historiens tout court. 
Cependant, on n'en a pas fini avec le profit qu'apporte la lecture de ce volume. Car ce qui caractérise Jean Rudhardt, c'est la hauteur d'où il prend les choses, dans le temps même où il descend dans le détail des textes. Il ne semble pas douteux que l'un des apports majeurs de ce livre doive concerner d'abord la pensée religieuse, par le biais de ses rapports avec le mythe. De fait, ce volume pourrait servir d'illustration choisie aux différents thèmes de méditation annoncés dans le titre du recueil Du mytbe, de la religion grecque et de la comprébension d'autrui.. En effet Jean Rudhardt rencontre avec Thémis et ses filles un terrain d'élection pour mettre en évidence que le mode d'expression mythique est consubstantiel à la pensée religieuse de la Grèce antique, et que l'ouverture à autrui en recueille un bénéfice important, puisqu'elle trouve à s'y développer selon deux directions : d'une part en ce que ce mode d'expression est aux antipodes d'une pensée dogmatique dont l'essence est de poser des bornes et de favoriser des replis frileux; de l'autre en ce que l'A. met à profit les rapports ou les oppositions unissant ou séparant la pensée moderne de la pensée antique pour nous faire pénétrer plus avant dans la compréhension de l'une comme de l'autre. Les enseignements principaux qu'on peut tirer de cette lecture sont en effet de divers ordres. Tout d'abord, naturellement, on y gagne une mise au clair de notions malaisées à appréhender; et l'on a fait un grand pas dans l'intelligence de Thémis, d'Eunomia, d'Eirènè ou de Dikè, quand on a saisi que toutes ont en commun de résider dans des principes qui régissent des institutions, plutôt que dans ces institutions elles-mêmes. Ensuite on retrouve, éclairé par un nouvel exemple, le sens des généalogies mythiques tel que Jean Rudhardt l'avait déjà mis en évidence par le passé. Il est à chercher dans un processus de spécification où les descendants réalisent dans leurs personnes les multiples virtualités contenues dans leurs parents : en l'occurrence, unie à Zeus Thémis conçoit des filles dotées de qualités qui furent les siennes, mais devenues plus efficaces, parce qu'elles sont mieux définies. Les caractères implicites et confondus en elle s'explicitent et se dissocient dans la personne de ses filles, toutes quatre restant unies entre elles par une communauté de nature. Enfin ce livre apporte une éclatante confirmation à un trait caractéristique de la religion grecque, qui la porte à concevoir une étroite solidarité entre la vie des cités et celle du cosmos : «L'appartenance de nos trois déesses au groupe des Hôrai, si proches de la croissance végétale, de la jeunesse et de la sexualité humaines, comme des rythmes sidéraux, alors qu'elles président en même temps à l'harmonie des sociétés humaines » (p. 152) en est un nouveau témoignage.

On voit donc que se conjuguent en ce livre plusieurs preuves de ce que l'expression mythique est inhérente à la pensée religieuse grecque. Ce mode d'expression intervient en effet pour donner une forme à la difficulté qu'éprouvaient les Grecs à conceptualiser des divinités qui font l'objet d'une aperception confuse. Jean Rudhardt s'en explique fort clairement : la prise de conscience d'une valeur et de ses exigences est l'expérience première et, avant toute réflexion, toute figuration même, cette aperception s'impose d'emblée à eux comme une réalité divine ( «es principes qui donnent de l'harmonie aux institutions, confèrent leur équilibre et leur efficacité aux comportements des hommes, les agents de cette heureuse disposition de l'âme et de la société sont tenus pour divins ", p. 100). Le langage traditionnel du mythe intervient pour permettre une élaboration de cette expérience. Aussi n'y a-t-il pas lieu de s'attarder aux différences de graphie qui prétendent (ne serait-ce que par commodité) séparer le nom commun du nom divin : les éditeurs modernes peuvent bien écrire les noms de Thémis et de ses filles tantôt avec une majuscule, tantôt avec une minuscule, "nous savons que ces procédés graphiques sont artificiels. Pour les Grecs, le nom ne change pas de sens » (p. 146-7). Cette constatation invite encore 
à reconsidérer les rapports du sacré et du profane, qui en Grèce se compénètrent totalement : dans le même texte, le nom de chacune de nos quatre entités ou déesses peut revêtir alternativement (voire simultanément) un sens profane et un sens divin, désigner une puissance impersonnelle et une figure anthropomorphe - autre signe que nos catégories ne sont pas universelles. C'est bien pourquoi il faut poursuivre le sentiment religieux d'une civilisation de la seule manière qui puisse permettre d'y accéder: de l'intérieur, comme Jean Rudhardt nous en montre le chemin pour la Grèce antique, en essayant toujours de retrouver l'expérience vécue, à laquelle il convient d'accorder la priorité. Elle seule permet de concevoir (ce qui semble si étrange à un esprit moderne quand il s'agit de religion grecque, et dont il n'y a cependant pas lieu de douter), qu'on puisse aller jusqu'à parler de foi : "Aux yeux des Grecs, la belle ordonnance de l'eunomia requiert les sanctions de la dikè. L'établissement de cette ordonnance, son maintien garanti par l'application de la justice, assurent la venue de la paix et sa permanence dans les cités humaines. La généalogie qui donne les trois déesses pour des sœurs indique une solidarité fondée sur des relations logiques dont on voudrait qu'elles fussent aussi des relations de fait. Le recours à l'expression mythique signifie que cette relation n'est pas simplement constatée dans la réalité de l'histoire mais qu'elle fait l'objet d'une espérance, si ce n'est d'une véritable foi » (p. 151-2). On constate à quel point cet ouvrage nous entraîne bien au-delà des perspectives qui nourrissent souvent les traités d'histoire des religions.

Un autre des grands points de la pensée de Jean Rudhardt illustré à neuf par ce livre réside dans la signification qu'il invite à reconnaître au polythéisme : il nous fait percevoir à quel point le langage mythique est adéquat pour exprimer l'aporie conceptuelle devant laquelle se trouvaient les Grecs quand ils entreprenaient d'évoquer la divinité. Quoique probablement sensibles à la prééminence éthique de l'unité, ils ne s'en sentaient pas moins aussi peu désireux qu'incapables d'enfermer le divin dans des concepts univoques et immuables ( $«$ Le divin échappe à l'intelligence humaine; il n'est pas possible d'en parler d'une façon claire, nul ne peut le faire avec autorité », p. 159). Le langage mythique leur a servi à suggérer, sans décrire d'une manière contraignante, sans définir ce qui répugne à être circonscrit, un divin dont les bienfaits divers peuvent être perçus comme à la fois solidaires et distincts. Ainsi l'exigence de justice est-elle sans doute « liée à l'action du dieu qui assure l'équilibre du monde et qui en garantit l'ordonnance » (p. 160). Mais il ne faüdrait pas, en faisant (comme Nilsson) de Dikè une simple personnification de la justice de Zeus, méconnaître "l'originalité de cette déesse, l'intensité de sa présence dans la conscience " (ibid.) d'Eschyle et dans celle de son public : il n'appartient pas aux modernes de rétablir un type de cohérence dont les anciens s'écartaient délibérément. Qu'on s'arrête aux textes d'Hésiode ou d'Eschyle par exemple, le langage dont ils usent, exprimant une intuition constante, délivrant un même enseignement à travers des formulations multiples et complémentaires, échappe au risque de donner à ce qui est insaisissable une clarté illusoire. Ces analyses pénétrantes obligent à une salutaire remise en perspective des implications du polythéisme : associé à l'expression mythique, il traduit un sentiment (que les idées reçues amènent d'ordinaire à méconnaître) de la transcendance du divin à l'égard des appellations et des figures divines. Il ne saurait être indifférent qu'il fasse partie de la religion grecque de n'imposer aucune orthodoxie.

Peut-être aura-t-on observé que ce compte-rendu fait peu de place à la discussion. C'est que l'A., toujours penché sur les textes (avec leur contexte, ce qui est rare), les commentant prudemment, n'offre guère de prise à la contestation: on n'a pas à peser la force de conclusions qui s'imposent avec l'évidence des faits. Est-il besoin de dire que si ce résultat provient, sans nul doute, de 
l'acuité d'esprit de l'A. et de sa modestie devant les textes, il est dû également au fait qu'il raisonne sur le grec ? C'est pourquoi hormis deux ou trois points qui n'affectent pas l'essentiel du propos, les réserves que la loi du genre m'impose d'exprimer portent sur des vétilles. Outre les inévitables coquilles, on relève deux probables erreurs de manipulation d'ordinateur (couper/copier/coller) qui amè nent à des redites (de la p. 105 à la p. 118, et de la p. 117 à la p.119), ainsi qu'un lapsus, p. 104 (où le nom Eurynomè mis pour Eunomiè laisse un instant perplexe). Trois points pourraient mériter d'être reconsidérés avant la seconde édition. À la p. 81, à propos de l'adjectif ăwoos, il pourrait être bienvenu d'invoquer toutes les malédictions funéraires dans lesquelles on voue un coupable à la

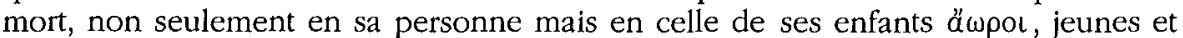
enlevés prématurément ( $c f$. L. Robert, Malédictions funéraires grecques, in CRAI, 1978 (2), 241-290 : p. 260 sq.). À la p. 117, référence est faite à la scène du procès figurée sur le bouclier d'Achille (Il. XVIII, 497-503), dans des termes qui ne prennent pas en considération la démonstration, pourtant probante à mon avis,

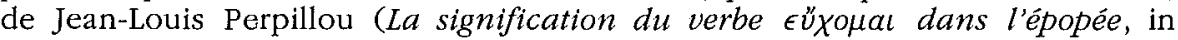
Mélanges de linguistique et de pbilologie grecques offerts à Pierre Chantraine, Paris, 1972, 169-182, p. 177 sq.); cette mise au point (qui aboutit pour les vers 48990 à la traduction: "L'un prétend se libérer entièrement par un versement..., l'autre refuse de rien recevoir ") entraîne des conséquences affectant non seulement le sens de $\epsilon \ddot{u} \chi 0 \mu a l$, mais encore le sens et la construction de ávalvoual. Par ailleurs on regrette l'absence d'index (en partic. locorum) d'autant plus vivement que la richesse du livre les rendrait plus précieux.

Mais ce sont là des remarques ponctuelles, sans conséquence sur l'utilité polyvalente de l'ouvrage, qui constitue à tant d'égards une leçon de méthode. Ce serait toutefois faire tort à ce maître livre que de le goûter surtout comme modèle. Plus important encore est l'intérêt qu'on y prend. Sa lecture constitue une nouvelle occasion d'apprécier les raisons pour lesquelles Jean Rudhardt fait autorité en matière de religion grecque.

Danièle Aubriot (Université d'Amiens)

Ileana Chirassi, Tullio Seppilur (éds), Sibille e Linguaggi oracolari: Mito, storia, tradizione. Atti del convegno internazionale di studi MacerataNorcia 20-24 Settembre 1994, Università degli Studi di Macerata, 1998 (PisaRoma, Istituti Editoriali e Poligrafici Internazionali, 1999), 1 vol. $17 \times 24 \mathrm{~cm}$, 822 p. (Icbnia. Collana del Dipartimento di Scienze Archeologiche e Storiche dell'Antichità, 3). ISBN : 88-8147-125-6.

La voix de la Sibylle se fait encore entendre, et cette fois à travers une enrichissante polyphonie. Il faut remercier I. Chirassi et T. Seppilli de leur effort pour publier les contributions du Colloque de Macerata-Norcia, qui nous permet maintenant de disposer d'une variété extraordinaire de points de vue et de méthodologies pour l'étude des aspects les plus divers de cette passionnante tradition. Il est périlleux de résumer le vaste contenu des 37 travaux du volume car il faut pour cela renoncer à détailler l'identité des auteurs et les titres des contributions : je ne citerai que les noms et un résumé des idées essentielles.

À noter l'importance de l'Introduction de Chirassi-Colombo (p. 13-35), qui ne se borne pas à résumer les travaux ici réunis, mais qui présente aussi une description du "parcours » (ou plutôt des parcours) de la Sibylle de l'Antiquité jusqu'au début du $x^{2}{ }^{e}$ siècle, pleine de stimulantes perspectives de travail. Ensuite, les contributions sont regroupées comme suit. 